\title{
Algebraic Extensions of Gaudin Models
}

\author{
Fabio MUSSO ${ }^{\dagger}$, Matteo PETRERA ${ }^{\ddagger}$ and Orlando RAGNISCO $\sharp$ \\ Dipartimento di Fisica E. Amaldi, Università degli Studi di Roma Tre \\ and \\ Istituto Nazionale di Fisica Nucleare, Sezione di Roma Tre \\ Via della Vasca Navale 84, 00146 Roma, Italy \\ $\dagger$ E-mail: musso@fis.uniroma3.it \\ ¥ E-mail: petrera@fis.uniroma3.it \\ \# E-mail: ragnisco@fis.uniroma3.it
}

This article is part of the special issue published in honour of Francesco Calogero on the occasion of his roth birthday

\begin{abstract}
We perform a Inönü-Wigner contraction on Gaudin models, showing how the integrability property is preserved by this algebraic procedure. Starting from Gaudin models we obtain new integrable chains, that we call Lagrange chains, associated to the same linear $r$-matrix structure. We give a general construction involving rational, trigonometric and elliptic solutions of the classical Yang-Baxter equation. Two particular examples are explicitly considered: the rational Lagrange chain and the trigonometric one. In both cases local variables of the models are the generators of the direct sum of $N \mathfrak{e}(3)$ interacting tops.
\end{abstract}

\section{Introduction}

In the present paper we are concerned with an extension of Gaudin model. Since it was introduced in the 70's the Gaudin model $[4,5]$ attracted considerable interest among theoretical and mathematical physicists, and played a distinguished role in the realm of integrable systems. Its peculariar properties, holding both at the classical and at the quantum level, are deeply connected with the long-range nature of the interaction described by its commuting Hamiltonians, which in fact yields a typical "mean field" dynamic.

The algebraic counterpart of these physical features lies in the linear $r$-matrix structure, that characterizes both the classical and the quantum model, and holds whatever be the dependence (rational, trigonometric, elliptic) upon the spectral parameter. The linear $r$-matrix structure does not depend on the site number $N$ (i.e. on the number of copies of the underlying Lie algebra) and moreover is not affected by a Inönü-Wigner contraction procedure. Such robustness property can for instance unable one to inherit the integrability property of the Lagrange top model from that of a two-site rational Gaudin magnet [10]. More generally, it allows one to construct, out of the $N$-site Gaudin model, novel interacting systems (both classical and quantum), whose underlying algebraic structure has been denoted in the literature as "jet extensions" of Lie algebras $[6,7]$. 
In this paper we will elucidate the connection between Lie algebra contractions and Lie algebra jet extensions in a general and systematic fashion, and we will derive the associated families of classical (and quantum) integrable models.

We have to mention here that pioneering results in this direction were obtained already in the 80's by Faddeev and Reshetikhin in their seminal paper [12], also, we have to mention the results obtained by Harnad in [7] for the rational case. However, we want to stress the connection among the systems investigated in the present paper and the Gaudin models, that to the best of our knowledge, is established here for the first time.

\section{Drinfel'd-Belavin solutions to cYBE and Gaudin models}

Let $\mathcal{P}$ be a Poisson manifold whose symplectic leaves have generically dimension $N$. Differentiable functions on $\mathcal{P}$ form a Poisson algebra and a completely integrable system on $\mathcal{P}$ is specified giving an abelian Poisson subalgebra of dimension $N$. In the modern theory of classical integrable systems the generators of this abelian Poisson subalgebra $\left\{H_{1}, \ldots, H_{N}\right\}$, called Hamiltonians of the system, are usually obtained as spectral invariants of a Lax matrix $L(\lambda)$ depending on a complex parameter $\lambda$ and whose matrix elements are functions on the Poisson manifold $\mathcal{P}$. Let us denote with $M$ the dimension of the square matrix $L(\lambda)$ defined by

$$
L(\lambda)=\sum_{i j} L_{i j}(\lambda) E_{i j}
$$

where $\left(E_{i j}\right)_{k l}=\delta_{i k} \delta_{j l}$ is the canonical basis of the $M \times M$ matrices. Introducing $\mathbb{1}$ as the identity matrix of dimension $M$ we define $\{L(\lambda) \otimes \mathbb{1}, \mathbb{1} \otimes L(\mu)\}$ as the matrix of Poisson brackets between the entries of $L(\lambda)$ :

$$
\{L(\lambda) \otimes \mathbb{1}, \mathbb{1} \otimes L(\mu)\} \doteq \sum_{i j, k l}\left\{L_{i j}(\lambda), L_{k l}(\mu)\right\} E_{i j} \otimes E_{k l} .
$$

In this context a theorem due to Babelon and Viallet [2] ensures that the commutativity of the Hamiltonians $\left\{H_{1}, \ldots, H_{N}\right\}$ is equivalent to the existence of a $M^{2} \times M^{2}$ matrix $r_{12}(\lambda, \mu)$ such that:

$$
\{L(\lambda) \otimes \mathbb{1}, \mathbb{1} \otimes L(\mu)\}=\left[r_{12}(\lambda, \mu), L(\lambda) \otimes \mathbb{1}\right]-\left[r_{21}(\mu, \lambda), \mathbb{1} \otimes L(\mu)\right],
$$

where

$$
r_{21}(\lambda, \mu)=\Pi r_{12}(\lambda, \mu) \Pi,
$$

with $\Pi$ the permutation operator on $\mathbb{C}^{M} \otimes \mathbb{C}^{M}$ :

$$
\Pi(x \otimes y)=y \otimes x .
$$

In this paper we will deal with an interesting subcase for equation (2.1) obtained when one imposes the following constraints on $r_{12}$ and $r_{21}$ :

$$
r_{12}(\lambda, \mu)=r_{12}(\lambda-\mu)=-r_{21}(\lambda-\mu),
$$


and moreover requires that the $r$-matrix be "not dynamical" (i.e. independent of phase space variables).

In this case equation (2.1) becomes (omitting the indices 12):

$$
\{L(\lambda) \otimes \mathbb{1}, \mathbb{1} \otimes L(\mu)\}+[r(\lambda-\mu), L(\lambda) \otimes \mathbb{1}+\mathbb{1} \otimes L(\mu)]=0 .
$$

It is well known that, in the case of a constant $r$-matrix, a sufficient condition for the r.h.s. of (2.2) to give rise to a well defined Poisson bracket is that $r$ satisfies the so-called "classical Yang-Baxter equation" (cYBE):

$$
\left[r_{13}(\lambda), r_{23}(\mu)\right]+\left[r_{12}(\lambda-\mu), r_{13}(\lambda)+r_{23}(\mu)\right]=0,
$$

where

$$
r_{12}=r \otimes \mathbb{1}, \quad r_{23}=\mathbb{1} \otimes r, \quad r_{13}=(\mathbb{1} \otimes \Pi) r_{12}(\mathbb{1} \otimes \Pi) .
$$

This equation was studied by Drinfel'd and Belavin [3] in the case when the $r$-matrix is an element of $\mathfrak{g} \otimes \mathfrak{g}$, with $\mathfrak{g}$ being a finite-dimensional simple Lie algebra. Once one has such a solution $r(\lambda)$ of equation (2.3), then $\left(\rho_{1} \otimes \rho_{2}\right) r(\lambda)$ with $\rho_{1}$ and $\rho_{2}$ faithful representations for $\mathfrak{g}$, gives a matrix solution for the cYBE.

Let $\left\{X^{\alpha}\right\}_{\alpha=1}^{\operatorname{dim} \mathfrak{g}}$ be a basis of the Lie algebra $\mathfrak{g}$, which is defined by the commutation relations

$$
\left[X^{\alpha}, X^{\beta}\right]=C_{\gamma}^{\alpha \beta} X^{\gamma}
$$

and let $g_{\alpha \beta}$ be the inverse of the Cartan-Killing metric related to this basis. Drinfel'd proved that under suitable non-degeneracy conditions on $r(\lambda)$ the only solutions to cYBE (2.3) are given by:

- rational solution: $r(\lambda)=\frac{g_{\alpha \beta} X^{\alpha} \otimes X^{\beta}}{\lambda}$,

- trigonometric solution: $r(\lambda)=\sum_{n=-\infty}^{\infty} \frac{\left(A^{n} \otimes \mathbb{1}\right) g_{\alpha \beta} X^{\alpha} \otimes X^{\beta}}{\lambda-n \omega}$,

- elliptic solution: $r(\lambda)=\sum_{n, m=-\infty}^{\infty} \frac{\left(A^{n} B^{m} \otimes \mathbb{1}\right) g_{\alpha \beta} X^{\alpha} \otimes X^{\beta}}{\lambda-n \omega_{1}-m \omega_{2}}, \quad[A, B]=0$.

Here $A$ and $B$ denote two finite order commuting automorphisms of $\mathfrak{g}$ not having a common fixed vector. Since $\mathfrak{s l}(n)$ is the only simple Lie algebra possessing such two automorphisms it follows that the elliptic solution can be defined only in the case $\mathfrak{g}=\mathfrak{s l}(n)$.

For our purposes, the relevant property of Drinfel'd solutions is that all of them can be generically written in the form:

$$
r(\lambda)=g_{\alpha \beta} X^{\alpha} \otimes X^{\beta} f^{\alpha}(\lambda)
$$

for suitable choices of the functions $f^{\alpha}(\lambda)$. 
From the cYBE we obtain a system of functional equations that must be satisfied by the functions $f^{\alpha}(\lambda)$. In fact the first member of (2.3) is given by

$$
\begin{aligned}
& {\left[g_{\alpha \beta} X^{\alpha} \otimes 1 \otimes X^{\beta} f^{\alpha}(\lambda), g_{\gamma \delta} 1 \otimes X^{\gamma} \otimes X^{\delta} f^{\gamma}(\lambda)\right]=} \\
& =g_{\alpha \beta} g_{\gamma \delta} C_{\eta}^{\beta \delta} X^{\alpha} \otimes X^{\gamma} \otimes X^{\eta} f^{\alpha}(\lambda) f^{\gamma}(\mu),
\end{aligned}
$$

while from the second one we obtain

$$
\begin{aligned}
& {\left[g_{\alpha \beta} X^{\alpha} \otimes X^{\beta} \otimes 1 f^{\alpha}(\lambda-\mu), g_{\gamma \eta}\left(X^{\gamma} \otimes 1 \otimes X^{\eta} f^{\gamma}(\lambda)+1 \otimes X^{\gamma} \otimes X^{\eta} f^{\gamma}(\mu)\right)\right]=} \\
& =g_{\alpha \beta} g_{\gamma \eta} f^{\alpha}(\lambda-\mu)\left[C_{\delta}^{\alpha \gamma} X^{\delta} \otimes X^{\beta} \otimes X^{\eta} f^{\gamma}(\lambda)+C_{\delta}^{\beta \gamma} X^{\alpha} \otimes X^{\delta} \otimes X^{\eta} f^{\gamma}(\mu)\right]= \\
& =g_{\alpha \beta} g_{\gamma \delta} X^{\alpha} \otimes X^{\gamma} \otimes X^{\eta} C_{\eta}^{\beta \delta}\left[f^{\delta}(\lambda-\mu) f^{\beta}(\lambda)-f^{\alpha}(\lambda-\mu) f^{\delta}(\mu)\right],
\end{aligned}
$$

where the last member is obtained from the previous one using the identity $g_{\gamma \eta} C_{\delta}^{\alpha \gamma}=$ $-g_{\gamma \delta} C_{\eta}^{\alpha \gamma}$ and swapping the indexes $\gamma \leftrightarrow \beta, \alpha \leftrightarrow \delta$ in the first term and $\gamma \leftrightarrow \delta$ in the second one.

Equating (2.5) and (2.6) we obtain the system of functional equations:

$$
\sum_{\beta, \delta=1}^{\operatorname{dim} \mathfrak{g}} g_{\alpha \beta} g_{\gamma \delta} C_{\eta}^{\beta \delta}\left[f^{\alpha}(\lambda) f^{\gamma}(\mu)+f^{\delta}(\lambda-\mu) f^{\beta}(\lambda)-f^{\alpha}(\lambda-\mu) f^{\delta}(\mu)\right]=0,
$$

for all $\alpha, \gamma, \eta=1, \ldots, \operatorname{dim} \mathfrak{g}$. Note that in equation (2.7) the summation is not on all the repeated indices, so that the sum is indicated explicitly.

To each matrix $r(\lambda)$ we can associate a Lax matrix whose entries are functions on the Lie-Poisson manifold $\mathcal{P}_{\mathfrak{g}}$ associated with $\mathfrak{g}$ in the following way: we choose a faithful representation $\rho$ of $\mathfrak{g}$ and we denote by $\left\{y^{\alpha}\right\}_{\alpha=1}^{\operatorname{dim} g}$ the coordinate functions related to the basis dual to $\left\{X^{\alpha}\right\}_{\alpha=1}^{\operatorname{dim} \mathfrak{g}}$ :

$$
\left[X^{\alpha}, X^{\beta}\right]=C_{\gamma}^{\alpha \beta} X^{\gamma} \quad \Longrightarrow \quad\left\{y^{\alpha}, y^{\beta}\right\}=C_{\gamma}^{\alpha \beta} y^{\gamma} .
$$

We define our Lax matrix as:

$$
L(\lambda)=\tau+g_{\alpha \beta} \rho\left(X^{\alpha}\right) y^{\beta} f^{\alpha}(\lambda),
$$

where $\tau \in \rho(\mathfrak{g})$ is a constant matrix chosen in such a way that

$$
[\tau \otimes \mathbb{1}+\mathbb{1} \otimes \tau, r(\lambda)]=0 .
$$

This Lax matrix satisfies the linear $r$-matrix algebra

$$
\{L(\lambda) \otimes \mathbb{1}, \mathbb{1} \otimes L(\mu)\}+[r(\lambda-\mu), L(\lambda) \otimes \mathbb{1}+\mathbb{1} \otimes L(\mu)]=0,
$$

with respect to the ordinary Lie-Poisson bracket, where $r$ is given by (2.4).

Now let us consider the Lie-Poisson manifold associated with the direct sum of $N$ copies of a simple Lie algebra $\mathfrak{g}$. We denote with $\left\{y_{i}^{\alpha}\right\}_{\alpha=1}^{\operatorname{dim} \mathfrak{g}}$ the coordinates on the dual of the $i$-th copy of $\mathfrak{g}$ :

$$
\left\{y_{i}^{\alpha}, y_{j}^{\beta}\right\}=\delta_{i j} C_{\gamma}^{\alpha \beta} y_{i}^{\gamma} \text {. }
$$


On each site we can define a matrix $L_{i}(\lambda)$ :

$$
L_{i}(\lambda)=g_{\alpha \beta} \rho\left(X^{\alpha}\right) y_{i}^{\beta} f^{\alpha}\left(\lambda-\lambda_{i}\right),
$$

where for any $i$ we have shifted the spectral parameter by a fixed quantity $\lambda_{i} \in \mathbb{C}$. Each matrix $L_{i}$ will obviously satisfy equation (2.2) and thanks to the locality of the Poisson relations (2.9) so will do also the total Lax matrix:

$$
L_{\mathcal{G}}(\lambda)=\tau+\sum_{i=1}^{N} L_{i}(\lambda) .
$$

It follows that the spectral invariants of the Lax matrix $L_{\mathcal{G}}(\lambda)$ will provide us with a set of involutive Hamiltonians that define the $N$-sites Gaudin model associated to $\mathfrak{g}$ $[4,5,13,9,11]$.

\section{Algebraic contractions of Gaudin models}

In the present section we illustrate our main result. Starting from Gaudin Lax operators (2.11) we obtain, through a Inönü-Wigner contraction, new Lax matrices satisfying the same $r$-matrix structure pertaining to the starting ones.

Let us recall the notion of generalized Inönü-Wigner contraction [8]. Let $V$ a complex finite, or infinite dimensional vector space. Let $\mathfrak{h}=(V, \mu)$ be a Lie algebra with Lie multiplication $\mu: V \times V \rightarrow V$.

The analytic notion of contraction can be described by a continuous family of homomorphism

$$
U(\epsilon): V \rightarrow V, \quad \epsilon \in(0,1], \quad U(1)=1,
$$

which are nonsingular for $\epsilon>0$ and singular for $\epsilon=0$.

The new Lie bracket on $V$,

$$
\mu_{\epsilon}(a, b)=U(\epsilon) \mu\left(U^{-1}(\epsilon) a, U^{-1}(\epsilon) b\right), \quad a, b \in V, \quad \epsilon>0,
$$

corresponds to a change of basis given by $U(\epsilon)$, and leads to the Lie algebra $\mathfrak{h}_{\epsilon}=\left(V, \mu_{\epsilon}\right)$ isomorphic to $\mathfrak{h}$. If

$$
\mu^{\prime}(a, b)=\lim _{\epsilon \rightarrow 0} \mu_{\epsilon}(a, b)
$$

exist for all $a, b \in V$, we call $\mathfrak{h}^{\prime}=\left(V, \mu^{\prime}\right)$ the contraction of $\mathfrak{h}$ by $U(\epsilon) ; \mathfrak{h}^{\prime}$ is, in general, not isomorphic to $\mathfrak{h}$.

For a generalized Inönü-Wigner contraction, $U(\epsilon)$ has a simple form, namely

$$
\begin{aligned}
& V=\bigoplus_{i=0}^{N} V^{(i)}, \quad N \geq 1, \\
& \left.U(\epsilon)\right|_{V^{(i)}}=\left.\epsilon^{n_{i}} \mathbb{1}\right|_{V^{(i)}},
\end{aligned}
$$


with

$$
0 \leq n_{0}<n_{1}<\ldots<n_{M}, \quad n_{i} \in \mathbb{R}, \quad i=0, \ldots, M .
$$

The necessary and sufficient condition for (3.1) to define a contraction is

$$
\mu\left(V^{(i)}, V^{(j)}\right) \subset \bigoplus_{l} V^{(l)},
$$

with

$$
n_{l} \leq n_{i}+n_{j}
$$

where all surviving structure constants are the same as for $\mathfrak{h}$. The special case $n_{0}=0$ and $N=1$ is called a simple Inönü-Wigner contraction.

We will apply a generalized Inönü-Wigner contraction to the direct sum of $N$ copies of a simple Lie algebra $\mathfrak{h}$ :

$$
\mathfrak{h}=\overbrace{\mathfrak{g} \oplus \cdots \oplus \mathfrak{g}}^{N} .
$$

The contraction procedure will be carried out directly on the Lie-Poisson algebra associated with $\mathfrak{h}$. We denote again with $\left\{y_{i}^{\alpha}\right\}_{\alpha=1}^{\operatorname{dim} \mathfrak{g}}$ the coordinates on the dual of the $i$-th copy of $\mathfrak{g}$, satisfying $\left\{y_{i}^{\alpha}, y_{j}^{\beta}\right\}=\delta_{i j} C_{\gamma}^{\alpha \beta} y_{i}^{\gamma}$ as in formula (2.9).

Since we need (3.2) we make the following change of basis in $\mathfrak{h}$ :

$$
\left(\begin{array}{c}
\tilde{y}_{0}^{\alpha} \\
\tilde{y}_{1}^{\alpha} \\
\vdots \\
\tilde{y}_{N-1}^{\alpha}
\end{array}\right)=\left(\begin{array}{cccc}
1 & 1 & \ldots & 1 \\
\nu_{1} & \nu_{2} & \ldots & \nu_{N} \\
\vdots & \vdots & \ddots & \vdots \\
\nu_{1}^{N-1} & \nu_{2}^{N-1} & \ldots & \nu_{N}^{N-1}
\end{array}\right)\left(\begin{array}{c}
y_{1}^{\alpha} \\
y_{2}^{\alpha} \\
\vdots \\
y_{N}^{\alpha}
\end{array}\right), \quad \alpha=1, \ldots, \operatorname{dim} \mathfrak{g}
$$

where the matrix defining the change of basis is a nonsingular Vandermonde matrix since we take $\nu_{j} \neq \nu_{k}, 1 \leq j \neq k \leq N$.

Note that in the new coordinates the equation (3.2) is satisfied if one identifies $V^{(i)}$ with the space generated by $\left\{\tilde{y}_{i}^{\alpha}\right\}_{\alpha=1}^{\operatorname{dim} \mathfrak{g}}$ and $n_{i}$ with $i$.

We define the homomorphism $U(\epsilon)$ by

$$
\left.U(\epsilon)\right|_{V^{(i)}}=\left.\epsilon^{i} \mathbb{1}\right|_{V^{(i)}} \quad i=1, \ldots, N-1 .
$$

We absorb the above homomorphism $U(\epsilon)$ into the definition of the new coordinates $\left\{\tilde{y}_{i}^{\alpha}\right\}_{\alpha=1}^{\operatorname{dim} \mathfrak{g}}$, namely hereafter we will denote

$$
\tilde{y}_{i}^{\beta} \doteq \sum_{j=1}^{N} \epsilon^{i} \nu_{j}^{i} y_{j}^{\beta} .
$$


The new bracket $\mu_{\epsilon}$ is defined by

$$
\begin{aligned}
\left\{\tilde{y}_{i}^{\alpha}, \tilde{y}_{j}^{\beta}\right\}_{\epsilon} & =\sum_{k, m=1}^{N} \epsilon^{i+j} \nu_{k}^{i} \nu_{m}^{j}\left\{y_{i}^{\alpha}, y_{j}^{\beta}\right\}= \\
& =C_{\gamma}^{\alpha \beta} \sum_{k=1}^{N} \epsilon^{i+j} \nu_{k}^{i+j} y_{k}^{\gamma}= \\
& =\left\{\begin{array}{cc}
C_{\gamma}^{\alpha \beta} \tilde{y}_{i+j}^{\gamma} & i+j<N, \\
O(\epsilon) & i+j \geq N .
\end{array}\right.
\end{aligned}
$$

After contraction we obtain the new Lie-Poisson algebra $\mathfrak{h}^{\prime}$ :

$$
\left\{\tilde{y}_{i}^{\alpha}, \tilde{y}_{j}^{\beta}\right\}=\left\{\begin{array}{cc}
C_{\gamma}^{\alpha \beta} \tilde{y}_{i+j}^{\gamma} & i+j<N \\
0 & i+j \geq N .
\end{array}\right.
$$

Let us give some remarks on the algebraic structure of our algebra $\mathfrak{h}^{\prime}$. If we define the new vector spaces

$$
\begin{aligned}
W^{(i)} & =\bigoplus_{j=i}^{N-1} V^{(j)} \quad i=0, \ldots, N-1, \\
W^{(0)} & \simeq \mathfrak{h}^{\prime},
\end{aligned}
$$

we obtain a flag of ideals

$$
\left\{W^{(i)}, W^{(j)}\right\} \subseteq W^{(j)} \quad j \geq i .
$$

In particular

$$
\left\{\mathfrak{h}^{\prime}, W^{(1)}\right\} \subseteq W^{(1)} .
$$

So we have an exact sequence:

$$
0 \longrightarrow W^{(1)} \stackrel{i}{\longrightarrow} \mathfrak{h}^{\prime} \stackrel{\pi}{\longrightarrow} \mathfrak{g} \longrightarrow 0
$$

where $i$ is the inclusion and $\pi$ is the projection on the quotient $\mathfrak{h}^{\prime} / W^{(1)}$; moreover we use the fact that $\mathfrak{h}^{\prime} / W^{(1)} \simeq V^{(0)} \simeq \mathfrak{g}$.

We can therefore see the contraction procedure as an extension of the starting Lie algebra $\mathfrak{g}$ that is known as the " $N$-th jet extension" of $\mathfrak{g}[6]$.

In the following theorem we give a recipe to extend the contraction procedure at the level of the equation (2.2).

Theorem 1. Let $\mathcal{L}(\lambda)$ be the Lax matrix on the Lie-Poisson algebra $\mathfrak{h}^{\prime}$ obtained from $L_{\mathcal{G}}(\lambda)$ (2.11) through the following procedure:

- make the identification $\lambda_{i}=-\epsilon \nu_{i}$, 
- take a formal power series expansion in $\epsilon$,

- take the limit $\epsilon \rightarrow 0$.

Then $\mathcal{L}(\lambda)$ satisfies

$$
\{\mathcal{L}(\lambda) \otimes \mathbb{1}, \mathbb{1} \otimes \mathcal{L}(\mu)\}+[r(\lambda-\mu), \mathcal{L}(\lambda) \otimes \mathbb{1}+\mathbb{1} \otimes \mathcal{L}(\mu)]=0,
$$

with respect to the Lie-Poisson algebra defined by

$$
\left\{\tilde{y}_{i}^{\alpha}, \tilde{y}_{j}^{\beta}\right\}=\left\{\begin{array}{cc}
C_{\gamma}^{\alpha \beta} \tilde{y}_{i+j}^{\gamma} & i+j<N \\
0 & i+j \geq N
\end{array}\right.
$$

with the same r-matrix given by (2.4).

Proof: Setting $\lambda_{i}=-\epsilon \nu_{i}$ in (2.11) we easily obtain the formal expansion

$$
L_{\mathcal{G}}(\lambda ; \epsilon)=\tau+\sum_{i=1}^{N} g_{\alpha \beta} \rho\left(X^{\alpha}\right) y_{i}^{\beta} \sum_{j=0}^{\infty} \frac{\left(\epsilon \nu_{i}\right)^{j}}{j !}\left(\frac{d}{d \lambda}\right)^{j} f^{\alpha}(\lambda) .
$$

Through the identification

$$
\tilde{y}_{i}^{\beta} \doteq \sum_{j=1}^{N} \epsilon^{i} \nu_{j}^{i} y_{j}^{\beta},
$$

we immediately get

$$
L_{\mathcal{G}}(\lambda ; \epsilon) \sim \tau+\sum_{i=0}^{N-1} \ell_{i}(\lambda) f_{i}^{\alpha}(\lambda)+O(\epsilon),
$$

where $\ell_{i}(\lambda)$ and $f_{i}^{\alpha}(\lambda)$ are given respectively by

$$
\begin{aligned}
& \ell_{i}(\lambda)=g_{\alpha \beta} \rho\left(X^{\alpha}\right) \tilde{y}_{i}^{\beta} . \\
& f_{i}^{\alpha}(\lambda)=\frac{1}{i !}\left(\frac{d}{d \lambda}\right)^{i} f_{0}^{\alpha}(\lambda), \quad f_{0}^{\alpha}(\lambda) \doteq f^{\alpha}(\lambda)
\end{aligned}
$$

Finally taking the limit $\epsilon \rightarrow 0$ we obtain:

$$
\mathcal{L}(\lambda)=\lim _{\epsilon \rightarrow 0} L_{\mathcal{G}}(\lambda ; \epsilon)=\tau+\sum_{i=0}^{N-1} \ell_{i}(\lambda) f_{i}^{\alpha}(\lambda) .
$$

Let us prove (3.3). We set $\tilde{y}_{i+j}^{\eta}=0$ if $i+j>N$. We have:

$$
\begin{aligned}
\{\mathcal{L}(\lambda) \otimes \mathbb{1}, \mathbb{1} \otimes \mathcal{L}(\mu)\} & =g_{\alpha \beta} g_{\gamma \delta} \rho\left(X^{\alpha}\right) \otimes \rho\left(X^{\gamma}\right) \sum_{i, j=0}^{N-1} f_{i}^{\alpha}(\lambda) f_{j}^{\gamma}(\mu)\left\{\tilde{y}_{i}^{\beta}, \tilde{y}_{j}^{\delta}\right\}= \\
& =g_{\alpha \beta} g_{\gamma \delta} \rho\left(X^{\alpha}\right) \otimes \rho\left(X^{\gamma}\right) C_{\eta}^{\beta \delta} \sum_{i, j=0}^{N-1} f_{i}^{\alpha}(\lambda) f_{j}^{\gamma}(\mu) \tilde{y}_{i+j}^{\eta}= \\
& =g_{\alpha \beta} g_{\gamma \delta} \rho\left(X^{\alpha}\right) \otimes \rho\left(X^{\gamma}\right) C_{\eta}^{\beta \delta} \sum_{i=0}^{N-1} \tilde{y}_{i}^{\eta} \sum_{j=0}^{i} f_{j}^{\alpha}(\lambda) f_{i-j}^{\gamma}(\mu) .
\end{aligned}
$$


On the other hand we have:

$$
\begin{aligned}
& {[r(\lambda-\mu), \mathcal{L}(\lambda) \otimes \mathbb{1}+\mathbb{1} \otimes \mathcal{L}(\mu)]=g_{\alpha \beta} g_{\gamma \eta} \times} \\
& \times\left[\rho\left(X^{\alpha}\right) \otimes \rho\left(X^{\beta}\right) f^{\alpha}(\lambda-\mu),\left(\rho\left(X^{\gamma}\right) \otimes \mathbb{1}\right) \sum_{i=0}^{N-1} \tilde{y}_{i}^{\eta} f_{i}^{\gamma}(\lambda)+\left(\mathbb{1} \otimes \rho\left(X^{\gamma}\right)\right) \sum_{i=0}^{N-1} \tilde{y}_{i}^{\eta} f_{i}^{\gamma}(\mu)\right]= \\
& =g_{\alpha \beta} g_{\gamma \eta} f^{\alpha}(\lambda-\mu) \times \\
& \times\left[C_{\delta}^{\alpha \gamma} \rho\left(X^{\delta}\right) \otimes \rho\left(X^{\beta}\right) \sum_{i=0}^{N-1} \tilde{y}_{i}^{\eta} f_{i}^{\gamma}(\lambda)+C_{\delta}^{\beta \gamma} \rho\left(X^{\alpha}\right) \otimes \rho\left(X^{\delta}\right) \sum_{i=0}^{N-1} \tilde{y}_{i}^{\eta} f_{i}^{\gamma}(\mu)\right]= \\
& =g_{\alpha \beta} g_{\gamma \delta} \rho\left(X^{\alpha}\right) \otimes \rho\left(X^{\gamma}\right) C_{\eta}^{\beta \delta} \sum_{i=0}^{N-1} \tilde{y}_{i}^{\eta}\left[f^{\delta}(\lambda-\mu) f_{i}^{\beta}(\lambda)-f^{\alpha}(\lambda-\mu) f_{i}^{\delta}(\mu)\right],
\end{aligned}
$$

where the last expression is obtained from the previous one using the identity $g_{\gamma \eta} C_{\delta}^{\alpha \gamma}=$ $-g_{\gamma \delta} C_{\eta}^{\alpha \gamma}$ and swapping the indexes $\gamma \leftrightarrow \beta, \alpha \leftrightarrow \delta$ in the first term and $\gamma \leftrightarrow \delta$ in the second one.

Considering equations (3.6) and (3.7) we obtain

$$
\begin{aligned}
\{\mathcal{L}(\lambda) & \otimes \mathbb{1}, \mathbb{1} \otimes \mathcal{L}(\mu)\}+[r(\lambda-\mu), \mathcal{L}(\lambda) \otimes \mathbb{1}+\mathbb{1} \otimes \mathcal{L}(\mu)]=g_{\alpha \beta} g_{\gamma \delta} \rho\left(X^{\alpha}\right) \otimes \rho\left(X^{\gamma}\right) \times \\
& \times C_{\eta}^{\beta \delta} \sum_{i=0}^{N-1} \tilde{y}_{i}^{\eta}\left[\sum_{j=0}^{i} f_{j}^{\alpha}(\lambda) f_{i-j}^{\gamma}(\mu)+f^{\delta}(\lambda-\mu) f_{i}^{\beta}(\lambda)-f^{\alpha}(\lambda-\mu) f_{i}^{\delta}(\mu)\right]
\end{aligned}
$$

Let us look in more detail at the quantity enclosed in the square brackets in equation (3.8). Using (3.5) we have:

$$
\begin{aligned}
\sum_{j=0}^{i} f_{j}^{\alpha}(\lambda) f_{i-j}^{\gamma}(\mu) & =\sum_{j=0}^{i} \frac{1}{j !}\left(\frac{d}{d \lambda}\right)^{j} f^{\alpha}(\lambda) \frac{1}{(i-j) !}\left(\frac{d}{d \mu}\right)^{i-j} f^{\gamma}(\mu)= \\
& =\frac{1}{i !} \sum_{j=0}^{i}\left(\begin{array}{l}
i \\
j
\end{array}\right)\left(\frac{d}{d \lambda}\right)^{j}\left(\frac{d}{d \mu}\right)^{i-j} f^{\alpha}(\lambda) f^{\gamma}(\mu)= \\
& =\frac{1}{i !}\left(\frac{d}{d \lambda}+\frac{d}{d \mu}\right)^{i} f^{\alpha}(\lambda) f^{\gamma}(\mu),
\end{aligned}
$$

and

$$
\begin{aligned}
f^{\delta}(\lambda-\mu) f_{i}^{\beta}(\lambda) & -f^{\alpha}(\lambda-\mu) f_{i}^{\delta}(\mu)= \\
& =\frac{1}{i !}\left[f^{\delta}(\lambda-\mu)\left(\frac{d}{d \lambda}\right)^{i} f^{\beta}(\lambda)-f^{\alpha}(\lambda-\mu)\left(\frac{d}{d \mu}\right)^{i} f^{\delta}(\mu)\right]= \\
& =\frac{1}{i !}\left(\frac{d}{d \lambda}+\frac{d}{d \mu}\right)^{i}\left[f^{\delta}(\lambda-\mu) f^{\beta}(\lambda)-f^{\alpha}(\lambda-\mu) f^{\delta}(\mu)\right] .
\end{aligned}
$$


Finally, from (3.9) and (3.10) we obtain

$$
\begin{aligned}
& \{\mathcal{L}(\lambda) \otimes \mathbb{1}, \mathbb{1} \otimes \mathcal{L}(\mu)\}+[r(\lambda-\mu), \mathcal{L}(\lambda) \otimes \mathbb{1}+\mathbb{1} \otimes \mathcal{L}(\mu)]=g_{\alpha \beta} g_{\gamma \delta} \rho\left(X^{\alpha}\right) \otimes \rho\left(X^{\gamma}\right) C_{\eta}^{\beta \delta} \times \\
& \quad \times \sum_{i=0}^{N-1} \tilde{y}_{i}^{\eta} \frac{1}{i !}\left(\frac{d}{d \lambda}+\frac{d}{d \mu}\right)^{i}\left[f^{\alpha}(\lambda) f^{\gamma}(\mu)+f^{\delta}(\lambda-\mu) f^{\beta}(\lambda)-f^{\alpha}(\lambda-\mu) f^{\delta}(\mu)\right]= \\
& =\sum_{i=0}^{N-1} \tilde{y}_{i}^{\eta} \frac{1}{i !}\left(\frac{d}{d \lambda}+\frac{d}{d \mu}\right)^{i} \times \\
& \quad \times\left\{g_{\alpha \beta} g_{\gamma \delta} \rho\left(X^{\alpha}\right) \otimes \rho\left(X^{\gamma}\right) C_{\eta}^{\beta \delta}\left[f^{\alpha}(\lambda) f^{\gamma}(\mu)+f^{\delta}(\lambda-\mu) f^{\beta}(\lambda)-f^{\alpha}(\lambda-\mu) f^{\delta}(\mu)\right]\right\},
\end{aligned}
$$

that vanishes thanks to equation (2.7). The fact that (3.4) is a Lie-Poisson algebra is then a plain consequence of (3.3).

\section{Extensions of $\mathfrak{s u}(2)$ Gaudin models: Lagrange chains}

In this section we will consider algebraic contractions of $\mathfrak{s u}(2)$ Gaudin models. We choose the matrices $\sigma^{1}, \sigma^{2}, \sigma^{3}$ as a basis of the fundamental representation of $\mathfrak{s u}(2)$ :

$$
\sigma^{1} \doteq \frac{\mathrm{i} \sigma_{x}}{2}=\frac{1}{2}\left(\begin{array}{cc}
0 & \mathrm{i} \\
\mathrm{i} & 0
\end{array}\right), \quad \sigma^{2} \doteq \frac{\mathrm{i} \sigma_{y}}{2}=\frac{1}{2}\left(\begin{array}{cc}
0 & 1 \\
-1 & 0
\end{array}\right), \quad \sigma^{3} \doteq \frac{\mathrm{i} \sigma_{z}}{2}=\frac{1}{2}\left(\begin{array}{cc}
\mathrm{i} & 0 \\
0 & -\mathrm{i}
\end{array}\right)
$$

where $\sigma_{x}, \sigma_{y}, \sigma_{z}$ are the Pauli matrices. In this basis the $r$-matrix (2.4) can be written in the form:

$$
r(\lambda)=-\sum_{\alpha=1}^{3} f_{0}^{\alpha}(\lambda) \sigma^{\alpha} \otimes \sigma^{\alpha}
$$

where

$$
\begin{aligned}
& f_{0}^{1}(\lambda)=\left\{\begin{array}{cl}
\frac{1}{\lambda} & \text { rational case } \\
\frac{1}{\sin \lambda} & \text { trigonometric case, } \\
\frac{\mathrm{dn}}{\operatorname{sn}}(\lambda) & \text { elliptic case }
\end{array}\right. \\
& f_{0}^{2}(\lambda)=\left\{\begin{array}{cl}
\frac{1}{\lambda} & \text { rational case } \\
\frac{1}{\sin \lambda} & \text { trigonometric case } \\
\frac{1}{\operatorname{sn}(\lambda)} & \text { elliptic case }
\end{array}\right. \\
& f_{0}^{3}(\lambda)=\left\{\begin{array}{cl}
\frac{1}{\lambda} & \text { rational case } \\
\cot \lambda & \text { trigonometric case } \\
\frac{\mathrm{cn}}{\operatorname{sn}}(\lambda) & \text { elliptic case. }
\end{array}\right.
\end{aligned}
$$


Let us consider the Lax matrix of the $\mathfrak{s u}(2)$ Gaudin magnet:

$$
L_{\mathcal{G}}(\lambda)=\tau+\sum_{i=1}^{N} \sum_{\alpha=1}^{3} \sigma^{\alpha} x_{i}^{\alpha} f_{0}^{\alpha}\left(\lambda-\lambda_{i}\right)
$$

where $\tau$ can be chosen as:

- any $\mathfrak{s u}(2)$-matrix in the rational case,

- $\sigma^{3}$ in the trigonometric case,

- the identity in the elliptic case,

and the local variables of the model $\left\{x_{i}^{\alpha}\right\}, \alpha=1,2,3, i=1, \ldots, N$ are the generators of the direct sum of $N \mathfrak{s u}(2)$ spins with the following Lie-Poisson brackets:

$$
\left\{x_{i}^{\alpha}, x_{j}^{\beta}\right\}=\delta_{i j} x_{i}^{\gamma} \quad i, j=1, \ldots, N
$$

where $\alpha \beta \gamma$ is the cyclic permutation of 123 .

The first natural extension of Gaudin models is obtained by contracting two copies of the Lie-Poisson algebra $\mathfrak{s u}(2)(N=2$ in the Lax matrix (4.5)), namely

$$
\mathfrak{s u}(2) \oplus \mathfrak{s u}(2) \simeq \mathfrak{o}(4) \rightarrow \mathfrak{e}(3)
$$

where $\mathfrak{e}(3)$ is the real euclidean Lie-Poisson algebra in the 3-space. The Lie-Poisson brackets of $\mathfrak{e}(3)$ are given by

$$
\left\{y^{\alpha}, y^{\beta}\right\}=y^{\gamma}, \quad\left\{y^{\alpha}, z^{\beta}\right\}=z^{\gamma}, \quad\left\{z^{\alpha}, z^{\beta}\right\}=0, \quad \alpha, \beta, \gamma=1,2,3 .
$$

After contraction we get the new Lax matrix:

$$
L(\lambda)=\tau+\sum_{\alpha=1}^{3} \sigma^{\alpha}\left[f_{0}^{\alpha}(\lambda) y^{\alpha}+f_{1}^{\alpha}(\lambda) z^{\alpha}\right]
$$

where $f_{0}^{\alpha}(\lambda)$ is given by $(4.2),(4.3),(4.4)$ and $f_{1}^{\alpha}(\lambda)$ is given by (3.5). Note that in the rational case the Lax matrix (4.6) is exactly the Lax matrix of the Lagrange top [1, 11]. This particular contraction procedure on the two-site $\mathfrak{s u}(2)$ Gaudin magnet has been considered in [10].

Moreover we observe that the spectral invariants of the Lax matrix (4.6) are the same for the rational, trigonometric and elliptic cases. However this is no more true if we extend our Lax matrix (4.6) to the $N$-bodies case as we did for Gaudin model (see equations (2.8),(2.10),(2.11)). Namely we consider the Lax matrix associated to the Lie-Poisson algebra of the direct sum of $N$ copies of $\mathfrak{e}(3)$ :

$$
\mathcal{L}(\lambda)=\tau+\sum_{i=1}^{N} \sum_{\alpha=1}^{3} \sigma^{\alpha}\left[f_{0}^{\alpha}\left(\lambda-\lambda_{i}\right) y_{i}^{\alpha}+f_{1}^{\alpha}\left(\lambda-\lambda_{i}\right) z_{i}^{\alpha}\right] .
$$




\subsection{The rational Lagrange chain}

The rational $\bigoplus_{i=1}^{N} \mathfrak{e}_{i}(3)$ chain, that we call rational Lagrange chain is derived from the following $2 \times 2$ Lax matrix

$$
\mathcal{L}(\lambda)=w \sigma^{3}+\sum_{i=1}^{N} \sum_{\alpha=1}^{3} \sigma^{\alpha}\left[\frac{y_{i}^{\alpha}}{\lambda-\lambda_{i}}+\frac{z_{i}^{\alpha}}{\left(\lambda-\lambda_{i}\right)^{2}}\right],
$$

where $\lambda_{i} \in \mathbb{C}$ and $w \in \mathbb{R}$ are the parameters of the model and $\lambda \in \mathbb{C}$ is the spectral parameter. The parameter $w$ denotes the intensity of an external field, taken as uniform (along the chain) and constant (in time).

Local variables of the model are generators of the direct sum of $N \mathfrak{e}(3)$ tops, $y_{i}^{\alpha}, z_{i}^{\alpha}$, $i=1, \ldots, N, \alpha=1,2,3$ with the following Lie-Poisson brackets:

$$
\left\{y_{i}^{\alpha}, y_{j}^{\beta}\right\}=\delta_{i j} y_{j}^{\gamma}, \quad\left\{y_{i}^{\alpha}, z_{j}^{\beta}\right\}=\delta_{i j} z_{j}^{\gamma}, \quad\left\{z_{i}^{\alpha}, z_{j}^{\beta}\right\}=0,
$$

where $\alpha \beta \gamma$ is the cyclic permutation of 123 .

The Lie-Poisson brackets (4.8) have $2 N$ Casimir functions:

$$
C_{i}^{(1)}=\sum_{\alpha=1}^{3} y_{i}^{\alpha} z_{i}^{\alpha}, \quad C_{i}^{(2)}=\sum_{\alpha=1}^{3}\left(z_{i}^{\alpha}\right)^{2} .
$$

As we have shown in the previous section the Lax matrix (4.7) satisfies the linear $r$-matrix algebra

$$
\{\mathcal{L}(\lambda) \otimes \mathbb{1}, \mathbb{1} \otimes \mathcal{L}(\mu)\}+[r(\lambda-\mu), \mathcal{L}(\lambda) \otimes \mathbb{1}+\mathbb{1} \otimes \mathcal{L}(\mu)]=0,
$$

where $\mathbb{1}$ is the $2 \times 2$ identity matrix and the $r$-matrix is given by

$$
r(\lambda)=-\frac{1}{\lambda} \sum_{\alpha=1}^{3} \sigma^{\alpha} \otimes \sigma^{\alpha} .
$$

The spectral curve $\Gamma$,

$$
\Gamma: \operatorname{det}(\mathcal{L}(\lambda)-\mu \mathbb{1})=0,
$$

is an hyperelliptic curve of genus $g=2 N-1$, given by

$$
-\mu^{2}=w^{2}+\sum_{i=1}^{N} \frac{R_{i}}{\lambda-\lambda_{i}}+\frac{S_{i}}{\left(\lambda-\lambda_{i}\right)^{2}}+\frac{C_{i}^{(1)}}{\left(\lambda-\lambda_{i}\right)^{3}}+\frac{C_{i}^{(2)}}{\left(\lambda-\lambda_{i}\right)^{4}},
$$

with the Hamiltonians $R_{i}$ and $S_{i}$ given by:

$$
\begin{aligned}
R_{i}= & w y_{i}^{3}+\sum_{k \neq i}^{N}\left(\frac{y_{i}^{1} y_{k}^{1}+y_{i}^{2} y_{k}^{2}+y_{i}^{3} y_{k}^{3}}{\lambda_{i}-\lambda_{k}}+\right. \\
& \left.+\frac{y_{i}^{1} z_{k}^{1}+y_{i}^{2} z_{k}^{2}+y_{i}^{3} z_{k}^{3}-y_{k}^{1} z_{i}^{1}-y_{k}^{2} z_{i}^{2}-y_{k}^{3} z_{i}^{3}}{\left(\lambda_{i}-\lambda_{k}\right)^{2}}-2 \frac{z_{i}^{1} z_{k}^{1}+z_{i}^{2} z_{k}^{2}+z_{i}^{3} z_{k}^{3}}{\left(\lambda_{i}-\lambda_{k}\right)^{3}}\right), \\
S_{i}= & \frac{1}{2}\left[\left(y_{i}^{1}\right)^{2}+\left(y_{i}^{2}\right)^{2}+\left(y_{i}^{3}\right)^{2}\right]+w z_{i}^{3}+ \\
& +\sum_{k \neq i}^{N}\left(\frac{y_{k}^{1} z_{i}^{1}+y_{k}^{2} z_{i}^{2}+y_{k}^{3} z_{i}^{3}}{\lambda_{i}-\lambda_{k}}+\frac{z_{i}^{1} z_{k}^{1}+z_{i}^{2} z_{k}^{2}+z_{i}^{3} z_{k}^{3}}{\left(\lambda_{i}-\lambda_{k}\right)^{2}}\right) .
\end{aligned}
$$


These are integrals of motion of the rational Lagrange chain, which are Poisson commuting:

$$
\left\{R_{i}, R_{j}\right\}=\left\{S_{i}, S_{j}\right\}=\left\{R_{i}, S_{j}\right\}=0, \quad i, j=1, \ldots, N .
$$

Notice that there is one linear integral given by

$$
\sum_{i=1}^{N} R_{i}=w \sum_{i=1}^{N} y_{i}^{3}
$$

Let us remark that if $N=1$ the Lagrange chain degenerates into the well-known $\mathfrak{e}(3)$ Lagrange top, whose Hamiltonians are given by $y^{3}$ and $\left(y^{1}\right)^{2}+\left(y^{2}\right)^{2}+\left(y^{3}\right)^{2}+2 w z^{3}$.

We can bring the curve $\Gamma$ into the canonical form by the scaling

$$
\mu \longmapsto \hat{\mu}=\mu \prod_{i=1}^{N}\left(\lambda-\lambda_{i}\right)^{2}
$$

The equation of the spectral curve becomes

$$
\begin{aligned}
-\hat{\mu}^{2} & =\left[w^{2}+\sum_{i=1}^{N} \frac{R_{i}}{\lambda-\lambda_{i}}+\frac{S_{i}}{\left(\lambda-\lambda_{i}\right)^{2}}+\frac{C_{i}^{(1)}}{\left(\lambda-\lambda_{i}\right)^{3}}+\frac{C_{i}^{(2)}}{\left(\lambda-\lambda_{i}\right)^{4}}\right] \prod_{i=1}^{N}\left(\lambda-\lambda_{i}\right)^{4}= \\
& =w^{2} \lambda^{4 N}+s_{1} \lambda^{4 N-1}+s_{2} \lambda^{4 N-2}+\ldots+s_{4 N}
\end{aligned}
$$

where the coefficients $s_{j}, j=1, \ldots, 4 N$ are linear combinations of the Hamiltonians and of the Casimir functions.

\subsection{The trigonometric Lagrange chain}

The trigonometric $\bigoplus_{i=1}^{N} \mathfrak{e}_{i}(3)$ chain, that we call trigonometric Lagrange chain, is derived from the following $2 \times 2$ Lax matrix

$$
\mathcal{L}(\lambda)=w \sigma^{3}+\sum_{i=1}^{N} \sum_{\alpha=1}^{3} \sigma^{\alpha}\left[f_{0}^{\alpha}\left(\lambda-\lambda_{i}\right) y_{i}^{\alpha}+f_{1}^{\alpha}\left(\lambda-\lambda_{i}\right) z_{i}^{\alpha}\right]
$$

where

$$
\begin{array}{lll}
f_{0}^{1}(\lambda)=\frac{1}{\sin \lambda}, & f_{0}^{2}(\lambda)=\frac{1}{\sin \lambda}, & f_{0}^{3}(\lambda)=\cot \lambda, \\
f_{1}^{1}(\lambda)=\frac{\cot \lambda}{\sin \lambda}, & f_{1}^{2}(\lambda)=\frac{\cot \lambda}{\sin \lambda}, & f_{1}^{3}(\lambda)=\cot ^{2} \lambda+1 .
\end{array}
$$

The Lax matrix (4.12) satisfies the linear $r$-matrix algebra (4.9) with $r$-matrix given by (4.1,4.3).

The spectral curve $\Gamma$,

$$
\Gamma: \quad \operatorname{det}(\mathcal{L}(\lambda)-\mu \mathbb{1})=0,
$$

is given by

$$
-\mu^{2}=Q+\sum_{i=1}^{N} \cot \left(\lambda-\lambda_{i}\right) R_{i}+\cot ^{2}\left(\lambda-\lambda_{i}\right) S_{i}+\cot ^{3}\left(\lambda-\lambda_{i}\right) C_{i}^{(1)}+\cot ^{4}\left(\lambda-\lambda_{i}\right) C_{i}^{(2)}
$$


where

$$
\begin{aligned}
Q= & w^{2}+\sum_{i=1}^{N}\left[2 w z_{i}^{3}+\left(y_{i}^{1}\right)^{2}+\left(y_{i}^{2}\right)^{2}\right]+ \\
& +2 \sum_{i=1}^{N} \sum_{k \neq i}^{N}\left\{\frac{1}{\sin \left(\lambda_{i}-\lambda_{k}\right)}\left[y_{k}^{1} z_{i}^{1}+y_{k}^{2} z_{i}^{2}+\cos \left(\lambda_{i}-\lambda_{k}\right) y_{k}^{3} z_{i}^{3}\right]+\right. \\
& \left.+\frac{2}{\sin ^{2}\left(\lambda_{i}-\lambda_{k}\right)}\left[\cos \left(\lambda_{i}-\lambda_{k}\right)\left(z_{k}^{1} z_{i}^{1}+z_{k}^{2} z_{i}^{2}\right)+z_{k}^{3} z_{i}^{3}\right]-y_{i}^{3} y_{k}^{3}\right\}, \\
R_{i}= & 2 w y_{i}^{3}+\left(z_{i}^{1}\right)^{2}+\left(z_{i}^{2}\right)^{2}+\left(z_{i}^{3}\right)^{2}+ \\
& +2 \sum_{k \neq i}^{N}\left\{\frac { 1 } { \operatorname { s i n } ( \lambda _ { i } - \lambda _ { k } ) } \left[y_{k}^{1} y_{i}^{1}+y_{k}^{2} y_{i}^{2}+\cos \left(\lambda_{i}-\lambda_{k}\right) y_{k}^{3} y_{i}^{3}-\right.\right. \\
& \left.-\left(\frac{2}{\sin ^{2}\left(\lambda_{i}-\lambda_{k}\right)}-1\right)\left(z_{k}^{1} z_{i}^{1}+z_{k}^{2} z_{i}^{2}\right)-\frac{2 \cos \left(\lambda_{i}-\lambda_{k}\right)}{\sin ^{2}\left(\lambda_{i}-\lambda_{k}\right)} z_{k}^{3} z_{i}^{3}\right]+ \\
& \left.+\frac{1}{\sin ^{2}\left(\lambda_{i}-\lambda_{k}\right)}\left[\cos \left(\lambda_{i}-\lambda_{k}\right)\left(y_{i}^{1} z_{k}^{1}+y_{i}^{2} z_{k}^{2}-y_{k}^{1} z_{i}^{1}-y_{k}^{2} z_{i}^{2}\right)+y_{i}^{3} z_{k}^{3}-y_{k}^{3} z_{i}^{3}\right]\right\}, \\
S_{i}= & \left(y_{i}^{1}\right)^{2}+\left(y_{i}^{2}\right)^{2}+\left(y_{i}^{3}\right)^{2}+2 w z_{i}^{3}+\left(z_{i}^{1}\right)^{2}+\left(z_{i}^{2}\right)^{2}+\left(z_{i}^{3}\right)^{2}+ \\
& +2 \sum_{k \neq i}^{N}\left\{\frac{1}{\sin \left(\lambda_{i}-\lambda_{k}\right)}\left[y_{k}^{1} z_{i}^{1}+y_{k}^{2} z_{i}^{2}+\cos \left(\lambda_{i}-\lambda_{k}\right) y_{k}^{3} z_{i}^{3}\right]+\right. \\
& \left.+\frac{1}{\sin ^{2}\left(\lambda_{i}-\lambda_{k}\right)}\left[\cos \left(\lambda_{i}-\lambda_{k}\right)\left(z_{k}^{1} z_{i}^{1}+z_{k}^{2} z_{i}^{2}\right)+z_{k}^{3} z_{i}^{3}\right]\right\} .
\end{aligned}
$$

Note that the coefficient $Q$ is functional dependent on the $S_{i}, R_{i}$ and $C_{i}^{(2)}$ :

$$
Q=w^{2}+\sum_{i=1}^{N} S_{i}-\left(\sum_{i=1}^{N} \frac{R_{i}}{2 w}\right)^{2}+\left(\sum_{i=1}^{N} \frac{C_{i}^{(2)}}{2 w}\right)^{2} .
$$

As in the rational case we identify in the coefficients $R_{i}, S_{i}$ the Poisson commuting integrals of the trigonometric Lagrange chain:

$$
\left\{R_{i}, R_{j}\right\}=\left\{S_{i}, S_{j}\right\}=\left\{R_{i}, S_{j}\right\}=0, \quad i, j=1, \ldots, N .
$$

\section{Concluding remarks}

In this paper we have performed a Inönü-Wigner contraction on Gaudin models, showing that the integrability property is preserved by this algebraic procedure. Other interesting structures can be inherited from the Gaudin models as well. For example in [10] it is shown how to obtain Bäcklund transformations for the Lagrange top from those of the $\mathfrak{s u}(2)$ Gaudin model. It turns out that it is possible to generalize this approach to the construction of Bäcklund transformations for Lagrange chains. This work is in progress and we plan to report the results in a forthcoming paper.

Finally we want to remark that the contraction procedure described in this paper can be applied to quantum Gaudin models as well, in a straightforward way. 


\section{Acknowledgments}

The authors wish to acknowledge the support from the GNFM (Gruppo Nazionale per la Fisica Matematica), research project: "Onde nonlineari, struttura tau e geometria delle varietà invarianti: il caso della gerarchia di Camassa-Holm".

\section{Appendix: The general rational Lax matrix}

In the proof of the theorem 3.1 we have seen that our Lax matrix $\mathcal{L}(\lambda)$ satisfies the $r$-matrix structure

$$
\{\mathcal{L}(\lambda) \otimes \mathbb{1}, \mathbb{1} \otimes \mathcal{L}(\mu)\}+[r(\lambda-\mu), \mathcal{L}(\lambda) \otimes \mathbb{1}+\mathbb{1} \otimes \mathcal{L}(\mu)]=0
$$

provided that the functions $f_{j}^{\alpha}(\lambda)$ satisfy the following system of functional equations (see equation (3.8)):

$$
\sum_{\beta, \delta=1}^{\operatorname{dim} \mathfrak{g}} g_{\alpha \beta} g_{\gamma \delta} C_{\eta}^{\beta \delta}\left[\sum_{j=0}^{i} f_{j}^{\alpha}(\lambda) f_{i-j}^{\gamma}(\mu)+f^{\delta}(\lambda-\mu) f_{i}^{\beta}(\lambda)-f^{\alpha}(\lambda-\mu) f_{i}^{\delta}(\mu)\right]=0,
$$

for all $\alpha, \beta, \gamma$ and $i=0, \ldots, N-1$.

In theorem 3.1 we have shown that the system (5.1) is satisfied with the choice (3.5); however this is not the general analytic solution to the system (5.1). In this appendix we give a conjecture about the general analytic solution of (5.1) in the rational case, where we have

$$
f_{0}^{\alpha}(\lambda)=\frac{1}{\lambda-c_{0}}
$$

$c_{0}$ being an arbitrary constant; $f_{0}^{\alpha}(\lambda)$ obviously does not depend on $\alpha$.

The system of functional equations (5.1) reduces to the following one:

$$
\sum_{i=0}^{k} f_{i}(\lambda) f_{k-i}(\mu)=\frac{f_{k}(\lambda)-f_{k}(\mu)}{\mu-\lambda} \quad k=0, \ldots, N .
$$

Our solution is based on the following algebraic conjecture:

Conjecture 1. Let $k \in \mathbb{N}, c_{1}, \ldots, c_{k}$ arbitrary constants and let us denote with $\left\{\vec{J}_{i}\right\}$ the set of vectors satisfying the Diophantine equation:

$$
\left\{\vec{J}_{i}\right\}=\left\{\vec{J} \in \mathbb{N}^{i}: J_{1}+2 J_{2}+\cdots+i J_{i}=i\right\},
$$

then the following polynomial identity holds:

$$
\sum_{i=0}^{k}\left(\sum_{\left\{\vec{J}_{i}\right\}} \frac{c_{1}^{J_{1}}}{J_{1} !} \ldots \frac{c_{i}^{J_{i}}}{J_{i} !} \lambda^{\left|\vec{J}_{i}\right|} \sum_{\left\{\vec{J}_{k-i}\right\}} \frac{c_{1}^{J_{1}}}{J_{1} !} \ldots \frac{c_{k-i}^{J_{k-i}}}{J_{k-i} !} \mu^{\left|\vec{J}_{k-i}\right|}\right)=\sum_{\left\{\vec{J}_{k}\right\}} \frac{c_{1}^{J_{1}}}{J_{1} !} \ldots \frac{c_{k}^{J_{k}}}{J_{k} !}(\lambda+\mu)^{\left|\vec{J}_{k}\right|},
$$

where we denoted with $\left|\vec{J}_{i}\right|$ the $1-$ norm of $\vec{J}_{i}$ :

$$
\left|\vec{J}_{i}\right|=\sum_{l=1}^{i} J_{l}
$$


Using the program for symbolic manipulation Maple we tested this conjecture for $N \leq$ 20.

Using the explicit form for the function $f_{0}(\lambda)$ and our conjecture it is not difficult to construct the general analytic solution for the system (5.2), reading:

$$
f_{i}(\lambda)=\sum_{\left\{\vec{J}_{i}\right\}} \frac{c_{1}^{J_{1}}}{J_{1} !} \ldots \frac{c_{i}^{J_{i}}}{J_{i} !}\left(\frac{d}{d \lambda}\right)^{\left|\vec{J}_{i}\right|} f_{0}(\lambda) \quad i=1, \ldots, N
$$

In fact, inserting (5.3) into (5.2) we get:

$$
\begin{aligned}
& \sum_{i=0}^{k} f_{i}(\lambda) f_{k-i}(\mu)= \\
& =\sum_{i=0}^{k}\left(\sum_{\left\{\vec{J}_{i}\right\}} \frac{c_{1}^{J_{1}}}{J_{1} !} \ldots \frac{c_{i}^{J_{i}}}{J_{i} !}\left(\frac{d}{d \lambda}\right)^{\left|\vec{J}_{i}\right|} f_{0}(\lambda) \sum_{\left\{\vec{J}_{k-i}\right\}} \frac{c_{1}^{J_{1}}}{J_{1} !} \ldots \frac{c_{k-i}^{J_{k-i}}}{J_{k-i} !}\left(\frac{d}{d \mu}\right)^{\left|\vec{J}_{k-i}\right|} f_{0}(\mu)\right)= \\
& =\sum_{\left\{\vec{J}_{k}\right\}} \frac{c_{1}^{J_{1}}}{J_{1} !} \ldots \frac{c_{k}^{J_{k}}}{J_{k} !}\left(\frac{d}{d \lambda}+\frac{d}{d \mu}\right)^{\left|\vec{J}_{k}\right|}\left(f_{0}(\lambda) f_{0}(\mu)\right)= \\
& =\sum_{\left\{\vec{J}_{k}\right\}} \frac{c_{1}^{J_{1}}}{J_{1} !} \ldots \frac{c_{k}^{J_{k}}}{J_{k} !}\left(\frac{d}{d \lambda}+\frac{d}{d \mu}\right)^{\left|\vec{J}_{k}\right|}\left(\frac{f_{0}(\lambda)-f_{0}(\mu)}{\mu-\lambda}\right)= \\
& =\frac{f_{k}(\lambda)-f_{k}(\mu)}{\mu-\lambda} .
\end{aligned}
$$

The fact that solutions (5.3) provide the general analytic solution to (5.2) can be argued taking the limit $\lambda \rightarrow \mu$ in the functional equations (5.2), yielding the system of ordinary differential equations:

$$
\sum_{i=0}^{k} f_{i}(\lambda) f_{k-i}(\lambda)=-\frac{d f_{k}(\lambda)}{d \lambda} \quad k=0, \ldots, N .
$$

As the system (5.4) is triangular, for any given $i$ the functions $f_{i}(\lambda)$ can be found solving a system of $i+1$ ordinary differential equations and therefore depend at most upon $i+1$ arbitrary parameters. This is exactly the number of arbitrary parameters entering our solutions (5.3).

Using our conjecture it is straightforward to extend the solutions (5.3) to the trigonometric and elliptic case. It is enough to replace $f_{i}(\lambda)$ with $f_{i}^{\alpha}(\lambda)$ and $f_{0}(\lambda)$ with the corresponding $f_{0}^{\alpha}(\lambda)$ given by $(4.2,4.3,4.4)$ :

$$
f_{i}^{\alpha}(\lambda)=\sum_{\left\{\vec{J}_{i}\right\}} \frac{c_{1}^{J_{1}}}{J_{1} !} \ldots \frac{c_{i}^{J_{i}}}{J_{i} !}\left(\frac{d}{d \lambda}\right)^{\left|\vec{J}_{i}\right|} f_{0}^{\alpha}(\lambda) \quad i=1, \ldots, N .
$$




\section{References}

[1] Audin M, Spinning tops, Cambridge University Press, 1996.

[2] Babelon O, Viallet C M, Hamiltonian structures and Lax equations, Phys. Lett. B237 (1990), 411-416.

[3] Belavin A A, Drinfel'd V G Solutions of the classical Yang-Baxter equation for simple Lie algebras, Funktsional. Anal. i Prilozhen. 16 (1982), 1-29, 96.

[4] Gaudin M, Diagonalisation d' une classe d' hamiltoniens de spin, J. de Physique 37 (1976), $1087-1098$.

[5] Gaudin M, La Fonction d' Onde de Bethe, Masson, Paris, 1983.

[6] Harnad J, Quantum isomonodromic deformations and the Knizhnik-Zamolodchikov equations, Symmetries and integrability of difference equations (Estérel, 1994) (1996), 155-161, CRM Proc. Lecture Notes, 9, Amer. Math. Soc., Providence, RI 155-161.

[7] Harnad J, Wisse M A, Moment Maps to Loop Algebras, Classical R-Matrix and Integrable Systems, in: Quantum Groups Integrable Models and Statistical Systems, World Scientific, Singapore, 1993, ed. J. Letourneux and L. Vinet, hep-th/9301104.

[8] Inönü E, Wigner E P, On the contraction of groups ant their representations, Proc. Natl Acad. Sci. 39 (1953), 510-24.

[9] Jurčo B, Classical Yang-Baxter equations and quantum integrable systems, Jour. Math. Phys. 30 (1989), 1289-1293.

[10] Kuznetsov V B, Petrera M, Ragnisco O, Separation of variables and Bäcklund transformations for the symmetric Lagrange top, Jour. Phys. A. 37 (2004), 8495-8512.

[11] Reyman A G, Semenov-Tian-Shansky M A, Group-Theoretical Methods in the Theory of Finite-Dimensional Integrable Systems, Dynamical Systems VII, Springer, 1994.

[12] Reshetikhin N Yu, Faddeev L D, Hamiltonian structures for integrable field theory models, Theor. Math. Phys. 56 (1983), 847-862 .

[13] Sklyanin E K, Separation of Variables in the Gaudin Model, Zap. Nauchn. Sem. Leningrad. Otdel. Mat. Inst. Steklov. (LOMI) 164 (1987), 47 (1989) , 2473-2488. 